\title{
ERRATUM
}

A. Messori - P. Becagli · S. Trippoli · E. Tendi

\section{Cost-effectiveness of adjuvant chemotherapy with cyclophosphamide + methotrexate + fluorouracil in patients with node-positive breast cancer}

In: Eur J Clin Pharmacol (1996) 51: 111-116

In the above article the sensitivity analysis described on page 114 (last paragraph) erroneously stated that the incremental cost-effectiveness ratio was $\$ 48$ per life year gained.

The correct value was $\$ 485$. 